\title{
Cloning, characterization and application of a glyceraldehyde-3-phosphate dehydrogenase promoter from Aspergillus terreus
}

\author{
Xuenian Huang $\cdot$ Xuefeng Lu $\cdot$ Jian-Jun Li
}

Received: 12 August 2013 / Accepted: 20 November 2013 / Published online: 4 December 2013

(C) Society for Industrial Microbiology and Biotechnology 2013

\begin{abstract}
It is important to develop native and highly efficient promoters for effective genetic engineering of filamentous fungi. Although Aspergillus terreus is an important industrial fungus for the production of itaconic acid and lovastatin, the available genetic toolbox for this microorganism is still rather limited. We have cloned the $5^{\prime}$ upstream region of the glyceraldehyde-3-phosphate dehydrogenase gene ( $g p d ; 2,150 \mathrm{bp}$ from the start codon) from A. terreus CICC 40205 and subsequently confirmed its promoter function using $s g f p$ (synthetic green fluorescent protein) as the reporter. The sequence of the promoter PgpdAt was further analysed by systematic deletion to obtain an effective and compact functional promoter. Two truncated versions of $\operatorname{PgpdAt}(1,081$ and $630 \mathrm{bp})$ were also able to drive $s g f p$ expression in A. terreus. The activities of these three PgpdAt promoters of varying different lengths were further confirmed by fluorescence, western blot and transcription. The shortest one (630 bp) was successfully applied as a driver of $v g b$ expression in the genetic engineering of $A$. terreus. The function of expressed haemoglobin was demonstrated by the $\mathrm{CO}$ (carbon monoxide)-difference spectrum and enhanced oxygen uptake rate, glucose consumption and itaconic acid titer. Our study was successful in developing and validating an efficient and compact native promoter for genetic engineering of A.terreus.
\end{abstract}

Electronic supplementary material The online version of this article (doi:10.1007/s10295-013-1385-0) contains supplementary material, which is available to authorized users.

X. Huang $\cdot$ X. Lu $\cdot$ J.-J. Li $(\bowtie)$

Key Laboratory of Biofuels, Shandong Provincial Key Laboratory of Energy Genetics, Qingdao Institute of Bioenergy and Bioprocess Technology, Chinese Academy of Sciences, No. 189 Songling Road, Qingdao 266101, China e-mail: lijj@qibebt.ac.cn
Keywords Aspergillus terreus · Glyceraldehyde-3phosphate dehydrogenase promoter - Native promoter . Promoter function · Vitreoscilla haemoglobin

\section{Introduction}

Aspergillus is an extremely large and diverse genus of filamentous fungi, and its members have long been used in biotechnology applications as cell factories for the production of a wide range of high value-added bioproducts, such as organic acids, pharmaceuticals and enzymes [1]. Among these, Aspergillus niger has been widely utilized for the production of citric acid, gluconic acid, amylase, glucoamylase, among others [1]. By contrast, A. terreus is a rather less known but still valuable species which has been commercially developed as an excellent itaconic acid producer; it is also used to produce lovastatin (Mevacor; Merck \& Co., Whitehouse Station, NJ) [2, 3]. Itaconic acid has been reported to be one of the most promising and flexible building blocks and is widely used as the monomer or comonomer in the manufacturing of plastics, resins and other industrial processes [2]. Lovastatin is an efficient cholesterol-lowering agent [3].

Advances in genetic engineering tools have resulted in the development of increasingly powerful approaches for genetic and metabolic engineering for improving the productivity of the target metabolites of filamentous fungi $[1,4]$. Improvement of the various components of genetic engineering, such as the promoters, the markers and the reporters, for a specific fungal host is a significant step towards developing the efficient and strong promoters required to achieve high-level expression of the target genes [5]. For fine-tuning of gene expression, some inducible promoters, including the glucoamylase 
promoter PglaA of A. niger [6], the Taka-amylase promoter PamyB of $A$. oryzae, among others, have been developed [7]. However, most of these usually require the strictly defined conditions for high- or low-level gene expression, respectively, and these are not suitable for industrial applications. Thus, the constitutive promoters, which are not dependent upon on the carbon or nitrogen source or the specific inducers, are preferred. For example, constitutive promoters, such as the pkiA promoter of the A. niger protein kinase A [8] and the glyceraldehyde3-phosphate dehydrogenase $(g p d)$ promoters, have been successfully used to express the respective heterologous genes in filamentous fungi [9-12]. Recently, many promoters from Aspergillus species have been cloned, characterized and applied in the genetic engineering of these species [13-15]. PgpdA from A. nidulans is by far the most frequently used promoter in fungal genetic engineering $[13,16]$.

Native promoters are the preferred promoters for genetic engineering of fungi because they are more efficient in directing gene expression than heterologous ones in some species of fungi $[9,13,17,18]$. Although A.terreus is an important producer of itaconic acid and lovastatin, the genetic tools for $A$. terreus, especially efficient native promoters, are lacking. PgpdA from A. nidulans is currently the most common promoter used for genetic engineering of $A$. terreus $[19,20]$. However, in order to prevent squelching or titration of the specific transcription factors, it is preferable to utilize multiple distinct promoters, with one specific promoter for each gene to be expressed. Multiple native promoters are therefore needed for genetic engineering of A.terreus. To date, the only investigated native promoter from $A$. terreus is the GlaAl (glucoamylase) promoter; however, it failed to drive expression of rotavirus nucleocapsid protein (VP6) in A. terreus [21].

It has recently been reported that the transcription level of the gpd gene (ATEG_09817.1) from A. terreus increased 15.8-fold under the optimal itaconic acid production conditions, suggesting that the gpd gene is regulated by the constitutive and highly active promoter [22]. This finding indicates that the promoter of the $g p d$ gene is a good candidate for developing a strong native one for $A$. terreus.

In the study reported here, we cloned the putative promoter region of the gpd gene from A. terreus. We then evaluated its promoter function and investigated the effects of promoter length on the promoter activity through the systematic deletion analysis. In order to demonstrate the potential of the $g p d$ promoter as a useful tool for genetic engineering, it was used for heterologous expression of the Vitreoscilla haemoglobin gene $(v g b)$ in A. terreus.

\section{Materials and methods}

Materials

Chemicals were obtained from Sigma (St. Louis, MO), oligonucleotides were synthesized by Shanghai Sangon Biotech Co. Ltd (Shanghai, China), and Taq and Pfu DNA polymerases and restriction endonucleases were from Fermentas/New England BioLabs (Ipswich, MA). The kits used for molecular cloning were from Omega Bio-tek Biotechnology (Norcross, GA). The vector pMD18-T-simple was obtained from Takara Biotechnology (Otsu, Japan), and the FastStart Universal SYBR Green Master ROX was obtained from Roche Applied Science (Penzberg, Germany). Trizol was from Invitrogen Life Technologies Corp. (Carlsbad, CA), hygromycin B was obtained from SolarbioScience Technology Co. Ltd (China), and mouse anti-GFP (green fluorescent protein) antibody and goat anti-mouse IgG-HRP (immunoglobulin G-horseradish peroxidase) were from TIANGEN Biotech (Beijing) Co. Ltd (Beijing, China).

Strains, plasmids, media and culture conditions

The plasmids pSGF957 and pWY1252, which were used for cloning the genes $s g f p, h p h, \operatorname{trpC}$ and $v g b$ from Vitreoscilla, were kindly provided by Professor Kim from Seoul National University and Professor Wen from China Agricultural University, respectively [10, 23]. Escherichia coli DH5 $\alpha$ was used for routine DNA transformation and plasmid isolation and was grown in Luria-Bertani broth at $37{ }^{\circ}$ C. A. terreus CICC 40205 was obtained from the China Center of Industrial Culture Collection and used in this study; it was grown on potato dextrose agar (PDA). Cultivation in shake flasks was carried out in itaconic acid production medium [IPM; g/L: glucose, $155 ; \mathrm{NH}_{4} \mathrm{NO}_{3}, 2$; $\left(\mathrm{NH}_{4}\right)_{2} \mathrm{HPO}_{4}, 0.2 ; \mathrm{MgSO}_{4}, 0.4 ; \mathrm{FeSO}_{4}, 0.02 ; \mathrm{ZnSO}_{4}, 0.04$; $\mathrm{CuSO}_{4}, 0.04$; corn steep, 1 ; pH 3.5].

Cloning of the gpd gene and the gpd promoters and construction of the expression cassettes

The primers used in this study are listed in Electronic Supplementary Material (ESM) Table S1. General molecular biology techniques were carried out following standard procedures [24]. The primers gpd-F/gpd-R for the gpd gene of A. terreus CICC 40205 were designed based on the annotation for ATEG_09817 in the genome of A. terreus NIH 2624 (http://www.cadre-genomes.org. uk/Aspergillus_terreus/Info/Index). The coding DNA sequence (CDS) and the full-length fragment of the gpd gene (ATEG_09817) were amplified by PCR using the cDNA and genomic DNA of A. terreus CICC 40205 as the 

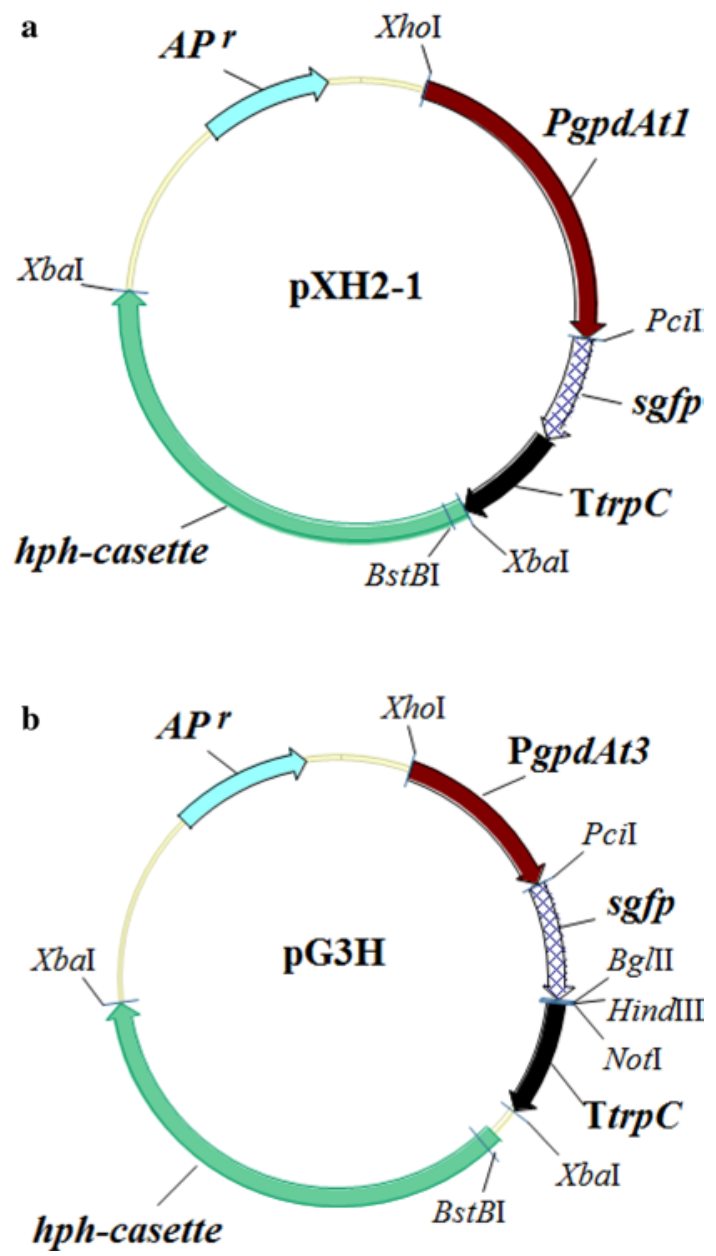

Fig. 1 Schematic structures of the expression vectors pXH2-1 (a) and $\mathrm{pG} 3 \mathrm{H}(\mathbf{b})$. hph-cassette Selection marker for hygromycin $\mathrm{B}$ resistance, Ttrpc Aspergillus nidulans trpC terminator, Pgpd promoter of glyceraldehyde-3-phosphate dehydrogenase ( $g p d)$ gene

respective templates. The terminator $\operatorname{trp} C$ from $A$. nidulans was used as the terminator in the current study $[5,10]$.

In order to cover all possible regulatory elements of the promoter of the gpd gene (ATEG_09817), we amplified and subsequently sequenced the upstream region of gpd (2,234 bp from the start codon), starting from the stop codon (TAA) of the upstream gene (ATEG_09816) and extending to the start codon (ATG) of the gpd gene, by PCR using genomic DNA of $A$. terreus CICC 40205 as the template and the primers PgpdAt-F/PgpdAt-R. The promoter PgpdAt1 (2,150 bp, full-length) was amplified by PCR using the primers PgpdAt1-F/PgpdAt-R1 and subsequently cloned into the vector pMD18-T-simple by TA cloning. The $P c i$ restriction site of the resulting vector was removed by site-directed mutagenesis using the primers mtpMD18F/mtpMD18-R, thus generating the plasmid pMD18msPgpdAt1. The sgfp-TtrpC fragment was amplified by PCR using the plasmid pSGF957 as the template and the primers
sgfp-F/TtrpC-R and then cloned into the vector pMD18msPgpdAt1 at the restriction sites of $P c i \mathrm{I}$ and $X b a \mathrm{I}$, thus creating the plasmid pMD18ms-PgpdAt1-sgfp. The $h p h$ cassette, which was cut from the plasmid pSGF957 with $X b a \mathrm{I}$, was inserted into the fragment pMD18ms-PgpdAt1-sgfp digested with $X b a \mathrm{I}$, resulting in construction of the plasmid pXH2-1 (Fig. 1a). The promoters PgpdAt2 (1,081 bp) and PgpdAt3 (630 bp) were amplified by PCR using the primer pairs PgpdAt2-F/PgpdAt-R1 and PgpdAt3-F/PgpdAt$\mathrm{R} 1$, respectively; these were then cloned into $\mathrm{pXH} 2-1$ at the restriction sites of $\mathrm{XhoI}$ and $P c i$ to give $\mathrm{pXH} 2-2$ and pXH2-3, respectively (Fig. 1a).

Construction of the Vitreoscilla haemoglobin expression cassette

The fragments $s g f p-A$ and $T \operatorname{trp} C-B$ were amplified by PCR from pXH2-1 using the primer pairs sgfp-F/sgfp$\mathrm{R} 1$ and TtrpC-F1/TtrpC-R1 respectively, then fused into the fragment $s g f p$-TtrpC containing the restriction sites of BglII, HindIII, and NotI by overlap extension PCR. The fragment $s g f p$-Ttrp $C-B$ obtained by digestion with $P c i$ I and BstBI was inserted into pXH2-3, which was then cut with $P c i$ and $B s t B I$ to generate the vector pG3H (Fig. 1b). The $v g b$ fragment was amplified by PCR from the plasmid pWY1252 using the primers vgb-F/vgb-R and then cloned into pGH3 at the restriction sites of PciI and $B g l I I$ to give the construct for $v g b$ expression. The $v g b$ gene was expressed under the control of the PgpdAt3 promoter.

Aspergillus terreus transformation

Mycelia from A. terreus CICC 40205 were grown in IPM at $30{ }^{\circ} \mathrm{C}$ for $16 \mathrm{~h}$ in shake flasks and then transformed using the protoplast-PEG method as previously described [13]. Transformants were selected on PDA-SH plates (PDA supplemented with $1.2 \mathrm{M}$ sorbitol and $100 \mathrm{mg} / \mathrm{L}$ hygromycin B). The integrations of the PgpdX::sgfp and $v g b$ cassettes into the genome of A.terreus were confirmed by genomic PCR using the primer pairs M13-47/sgfp-seqR1 and M13-47/vgb-R, respectively. Representative transformants were randomly selected for further analysis.

Evaluation of the Pgpd promoters

Quantitative real-time PCR (qRT-PCR) was performed using FastStart Universal SYBR Green Master ROX (Roche Applied Science) on a Light Cycler (Roche Applied Science) to estimate the transgene copy number of the selected transformants [5, 25]. The ATEG_02314 gene (a single copy in the genome of A. terreus NIH 2624) was used as the control. 
The bright field and fluorescent images of the selected transformants at the stages of conidia, young hyphae, and mature hyphae were observed by fluorescence microscopy (Olympus model BX51; Olympus, Tokyo, Japan). Young and mature hyphae were obtained by cultivation in shake flasks at $37{ }^{\circ} \mathrm{C}$ for 11 and $36 \mathrm{~h}$, respectively. The total proteins were extracted from mature hyphae ground with liquid nitrogen in solution (50 mM sodium phosphate, $\mathrm{pH} 7.2$, $1 \mathrm{mM}$ EDTA, $1 \mathrm{mM}$ PMSF). The protein concentration was determined by the Bradford method using bovine serum albumin as a standard and diluted to approximately $0.5 \mathrm{mg} /$ ml. The expressed GFP was also analysed by western blot following the standard protocol. Fluorescence intensity was measured using the Synergy HT Multi-Mode Microplate Reader (BioTek, Winooski, VT) at an excitation of $485 \mathrm{~nm}$, emission at $516 \mathrm{~nm}$, optics position at the top and sensitivity of 40 .

The functions of the promoters were also investigated at the transcription level of $s g f p$ by qRT-PCR [9]. The actin gene (ATEG_06973) was used as the endogenous control. qRT-PCR was performed according to the protocol provided by the manufacturer, and the data were analyzed as reported [26].

\section{Analysis of the $v g b$ transformants}

The fermentation processes of the parental strain and the best itaconic acid producer V08 were investigated by cultivation in 500-ml shake flasks every $12 \mathrm{~h}$. Each shake flask contained $55 \mathrm{ml}$ IPM and following inoculation with $2.5 \times 10^{7}$ spores the flasks were incubated for $72 \mathrm{~h}$ at $37{ }^{\circ} \mathrm{C}, 200 \mathrm{rpm}$. The initial $\mathrm{pH}$ was 3.25 and fell to around 2.6 by the end of the fermentation. Three independent experiments were set for each transformant. All assays were done at least in duplicate.

The activity of the expressed $\mathrm{VHb}$ was demonstrated by the CO-difference spectra of the cell-free extracts of V08 and the parental strain [20]. Total proteins were extracted from mycelia as described above.

Cells were harvested by centrifugation, washed with $100 \%$ oxygen-saturated IPM and resuspended in the same medium. The changes in dissolved oxygen were measured using the Oxylab Oxygen Monitoring System (Hansatech Instruments, Norfolk, UK).

Mycelial dry weight was determined as described previously [27].

Residual glucose was quantified using the biosensor (SBA-40C) from Biology Institute of Shandong Academy of Science (Jinan, China) following the standard protocol.

The itaconic acid produced was quantified by high-performance liquid chromatography using an Aminex HPX$87-\mathrm{H}$ column $(300 \times 7.8 \mathrm{~mm})$; detection was at $210 \mathrm{~nm}$. The column was operated at $35{ }^{\circ} \mathrm{C}$ with a mobile phase of
$4 \mathrm{mM} \mathrm{H}_{2} \mathrm{SO}_{4}$ at a flow rate of $0.6 \mathrm{ml} / \mathrm{min}$. Authentic itaconic acid was used as a standard to calculate the final concentration of itaconic acid produced.

\section{Results and discussion}

Cloning and sequence analysis of the gpd gene from A. terreus CICC 40205

The gpd gene was amplified by PCR using the cDNA of A. terreus CICC 40205 as the template and the primers gpd-F/gpd-R. However, the sequencing results showed different open reading frames from the annotated gpd (ATEG_09817) gene in the genome sequence of $A$. terreus NIH 2624, including the translation initiation site, the numbers and positions of the exons and introns. Based on our experimental results and sequence alignment of the gpd genes from other Aspergilli, including A. niger, $A$. nidulans, A. flavus, A. fumigatus and A. oryzae (data not shown), we corrected the CDS of the gpd gene of $A$. terreus CICC 40205, which has been deposited in GenBank under accession no. KC213825.

Cloning, sequence analysis and functional characterization of PgpdAtl

A 2,234-bp upstream sequence from the start codon (ATG) of the gpd gene of A. terreus CICC 40205 was cloned and sequenced; this sequence showed $93.8 \%$ identity with the annotated one for ATEG_09817 in the genome sequence of $A$. terreus NIH 2624. Sequence alignment of the upstream regions of the gpd genes from other Aspergilli was carried out (http://www. cadre-genomes.org.uk/index.html), and several conserved regions were observed (Fig. 2). The one located about -400 bp from the start codon (ATG, A as +1) has been referred to as the gpd box (Fig. 2) [11]. This gpd box is an important element in regulating transcription of the gpd gene, and its deletion was found to result in a $50 \%$ decrease in the expression level of the reporter gene coding for $\beta$-glucuronidase (gus) $[11,17]$. In contrast, introduction of the gpd box $A$ into the upstream of the amdS gene led to a 30-fold increase in expression of the reporter gene [28].

The sequence analysis revealed that Intron-1 (-94 to $-11 \mathrm{bp})$ was removed during the processing of mRNA (Fig. 2), which might not play a role or roles in the translation of the gpd gene. In an earlier study, deletion of this region in A. nidulans had no effect on the expression level of the reporter gene [11]. Therefore, we did not include Intron-1 in the amplification of the putative promoter PgpdAt1. 


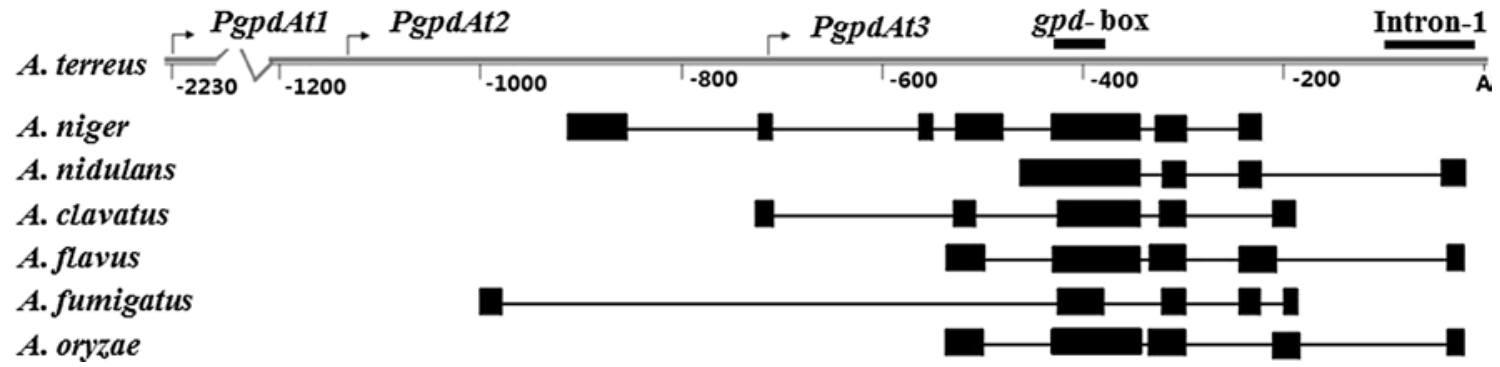

Fig. 2 Conserved regions in the putative promoters of the genes coding for the gpd gene from seven filamentous fungi. The GenBank accession numbers of the selected gpd genes are: Aspergillus niger CBS 513.88 (An16g01830); A. nidulans FGSC A4 (ANIA_08041);

A. flavus NRRL 3357 (AFLA_025100); A. fumigates Af293 (AFUA_5G01970); A. oryzae RIB40 (AO090003001322); A. clavatus NRRL1(ACLA_003290)
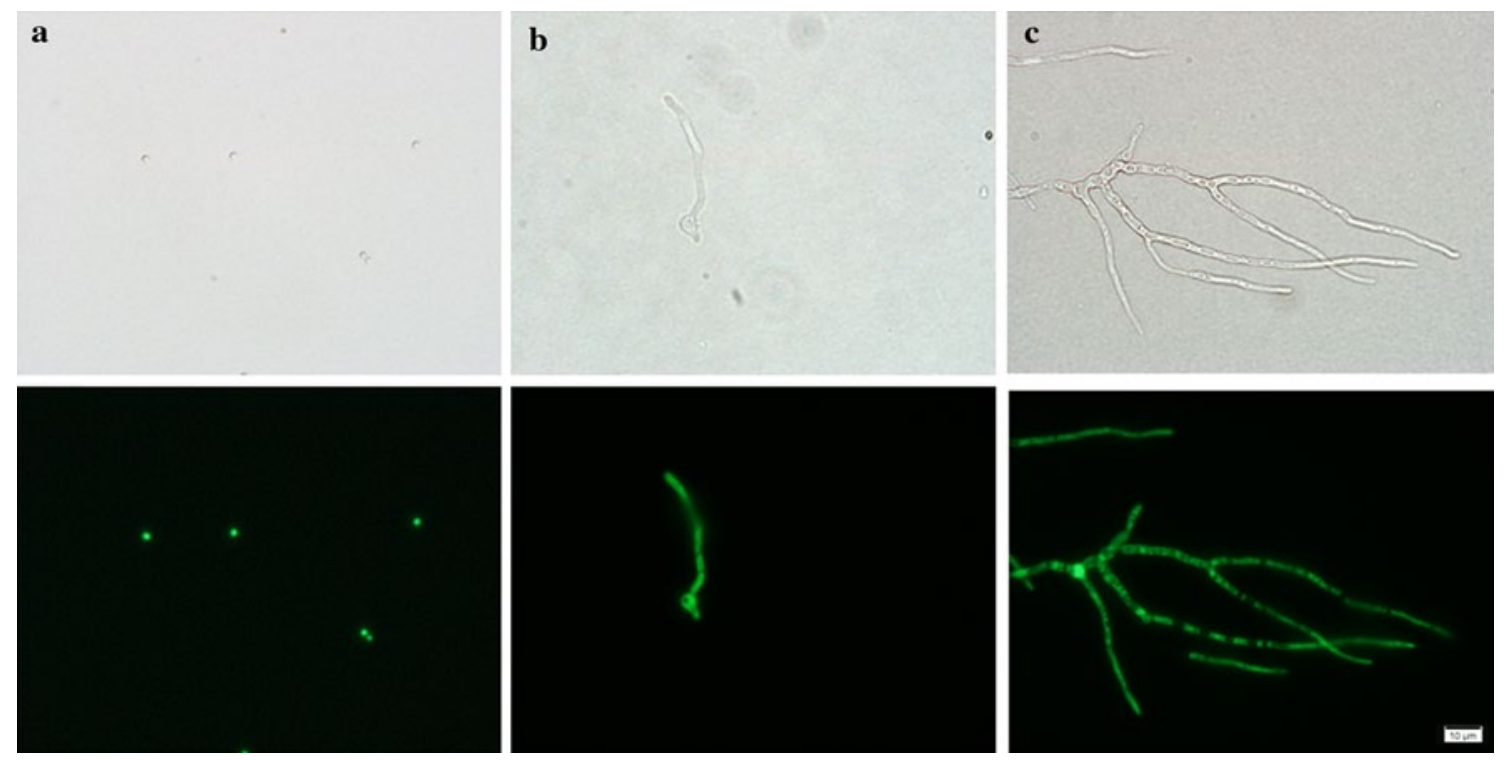

Fig. 3 Bright field and fluorescence images of A. terreus transformant 1-1 carrying the PgpdAt1::sgfp construct in different growth stages. a Conidia, b young hyphae, c mature hyphae. $s g f p$ Synthetic green fluorescence protein. Scale bar $10 \mu \mathrm{m}$

The promoter function of the putative promoter region was evaluated using $s g f p$ (synthetic GFP) as the reporter. The expression cassette pXH2-PgpdAt1 for the promoter PgpdAtl (2,150 bp, the full-length fragment of the gpd promoter) was constructed and expressed in A. terreus CICC 40205 (Fig. 1a). The integration of the PgpdAt1::sgfp cassette into the genome of $A$. terreus was confirmed by genomic PCR (ESM Fig. S1). The bright field and fluorescent images of randomly picked samples of A. terreus transformant 1-1 were taken at different stages: conidia, young hyphae and mature hyphae (Fig. 3). As a negative control, the parental strain A. terreus CICC 40205 did not show any visible GFP fluorescence at all three of these stages (data not shown). These results clearly demonstrate that PgpdAt1 successfully drove sgfp expression in A. terreus.

\section{Deletion analysis of PgpdAt1}

In order to obtain the efficient and compact promoters which are desirable in genetic engineering, we designed two truncated promoters, namely, PgpdAt2 (1,081 bp) and PgpdAt3 (630 bp), which we amplified by PCR (Fig. 2). PgpdAt3 contains the highly conserved regions, including the gpd box (Fig. 2). However, taking into account the possibility that PgpdAt3 might not cover all regulatory features, we also designed PgpdAt2, which includes all conserved regions and is 451 bp longer than PgpdAt3 (Fig. 2). The corresponding expression cassettes were constructed and expressed in $A$. terreus CICC 40205 . Interestingly, the truncated promoters PgpdAt2 and PgpdAt3 were also able to drive $s g f p$ expression in A. terreus at the conidia, young hyphae and mature hyphae stages (ESM Fig. S2 and Fig. S3). 

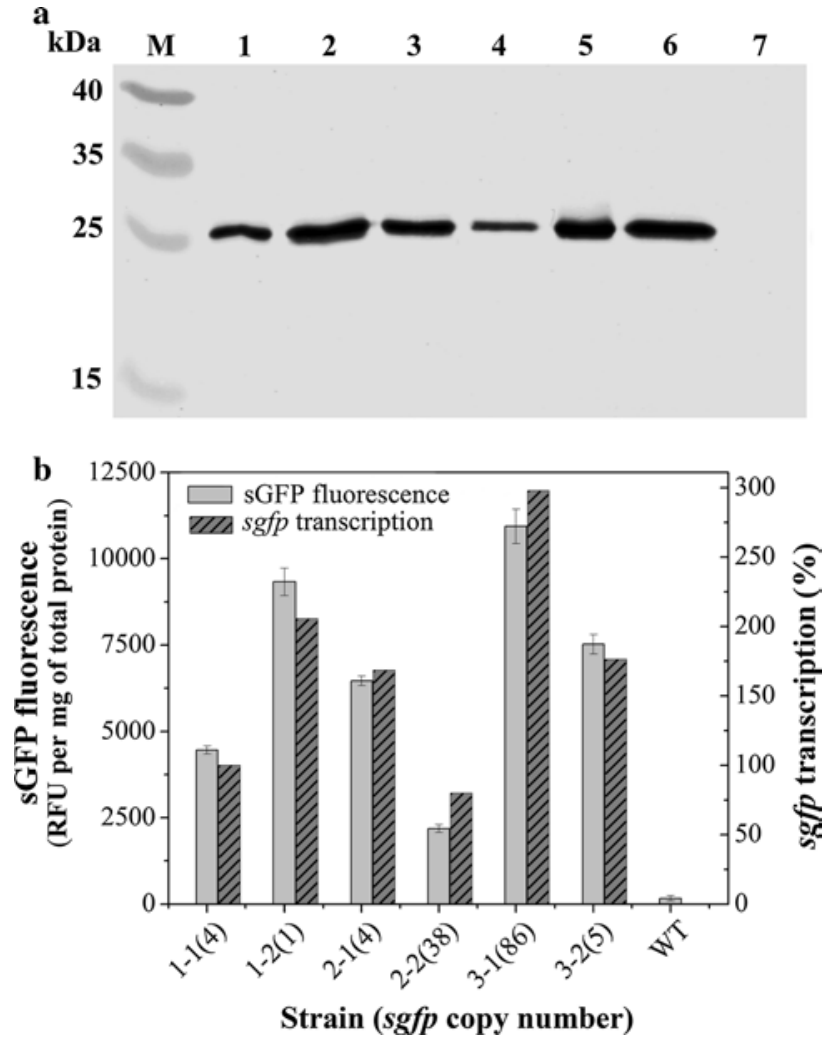

Fig. 4 Evaluation of the Pgpd promoters. a Western blot analysis of expressed GFP. Lanes: $M$ Protein marker, 1, 2 transformants 1-1 and 1-2, respectively, carrying the PgpdAt1::sgfp construct, 3, 4 transformants 2-1 and 2-2, respectively, harboring the PgpdAt2::sgfp construct, 5, 6 transformants 3-1 and 3-2, respectively, carrying the PgpdAt3::sgfp construct, 7 A. terreus CICC 40205. b Fluorescence and transcription analysis of GFP (copy number in parenthesis). The transcription level of the PgpdAt1 transformant 1-1 was set to $100 \%$

The promoter activities of these three PgpdAt promoters of different lengths were further demonstrated by western blot analysis (Fig. 4a), fluorescence studies (Fig. 4b), and transcription (Fig. 4b). These experiments gave consistent results and showed a similar trend (Fig. 4):

(1) The shortest promoter PgpdAt3, which includes the highly conserved regions, completely retained promoter activity.

(2) Deletion of the fragments $-2150 \mathrm{bp}$ to $-1081 \mathrm{bp}$ and $-2150 \mathrm{bp}$ to $-630 \mathrm{bp}$, respectively, did not result in reduced promoter activity, implying that the absence of positive regulatory elements located between -2150 bp and -630 bp (Figs. 2, 4). The efficient and compact native gpd promoter PgpdAt3 from A. terreus was identified. Some short and functional gpd promoters have been reported, including the 946-bp promoter from Metarhizium acridum [9], the 442-bp promoter from Lentinus edodes [29] and the 190-bp promoter from Candida bombicola [12]. It would appear that the gpd promoters from different microorganisms show different regulatory characteristics.

(3) Multicopy sgfp did not increase the translation/transcription level (Fig. 4b), possibly due to the titration of endogenous factors when multiple integrated copies are present [5]. However, it is still controversy on this possibility $[5,17]$.

(4) Two transformants for the same promoters exhibited different translation/transcription levels; for example, transformants 1-1 and 1-2 for PgpdAt1, transformants 2-1 and 2-2 for PgpdAt2. The difference might arise from the different integration sites, since there was no obvious relationship between copy number and translation/transcription, and the integrations were expected to be random in our study.

Application of PgpdAt in genetic engineering of A. terreus

Even a brief interruption of aeration during fermentation significantly decreases the itaconic acid titer [5]. Vitreoscilla haemoglobin ( $\mathrm{VHb}$ ) has been widely used to alleviate the adverse effects of hypoxic conditions and to improve growth, enzyme production and metabolism in various microorganisms [30, 31]. In order to demonstrate biotechnological application of PgpdAt as the promoters in genetic engineering of $A$. terreus, we expressed $v g b$ in A. terreus CICC 40205 under the control of the efficient and compact promoter PgpdAt3.

All hygromycin-resistant transformants were grown in IPM. The best itaconic acid producer, V08, was chosen from 27 transformants for further analysis. The activity of expressed $\mathrm{VHb}$ in A. terreus was demonstrated by the $\mathrm{CO}$ (carbon monoxide)-difference spectrum (Fig. 5). The peak we observed at $422 \mathrm{~nm}$ for V08 resulted from CO binding to $\mathrm{VHb}$, whereas a corresponding peak was not observed in

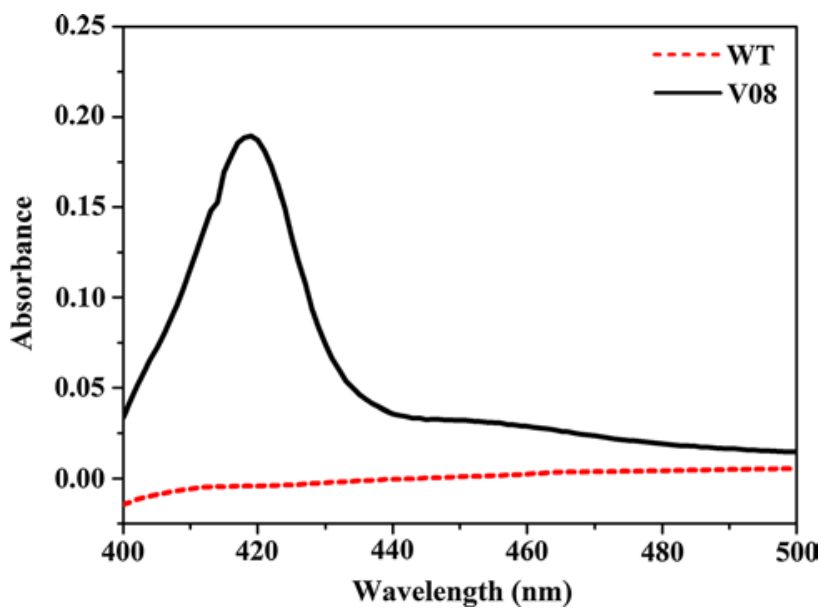

Fig. 5 Carbon monoxide difference spectra of cell-free extracts of transformant V08 and the wild type (WT) 

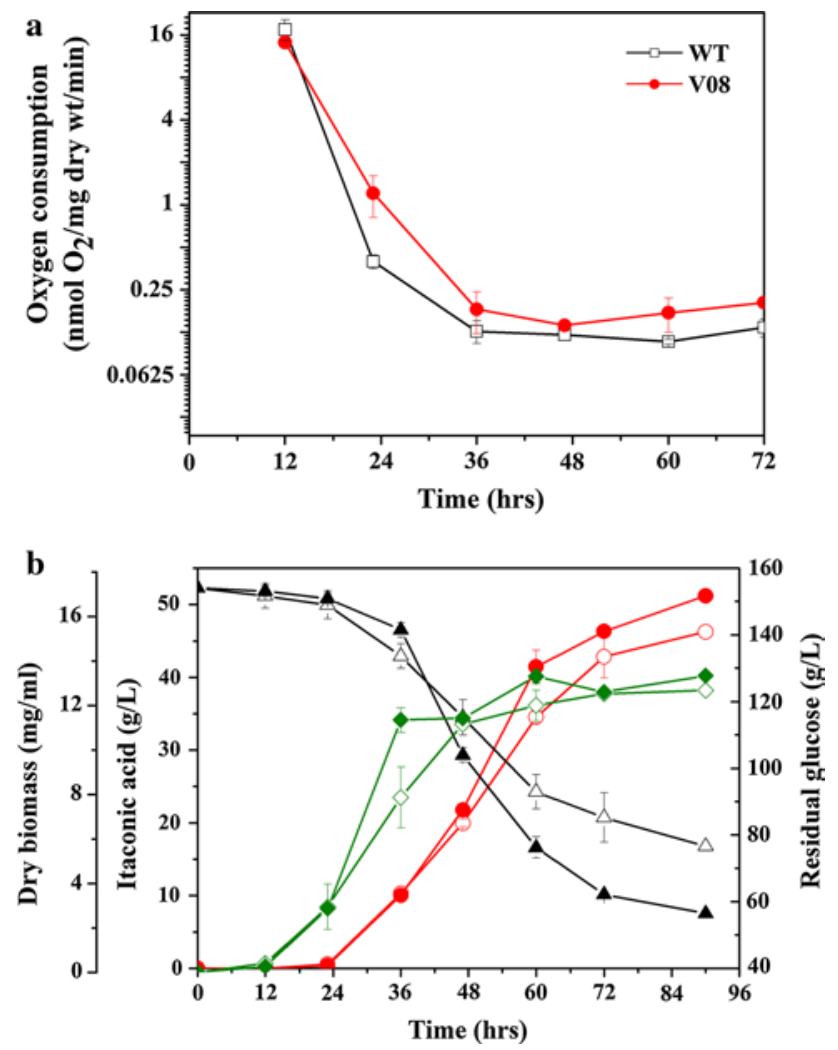

Fig. 6 a Oxygen uptake in transformant V08 and WT. b Time courses of dry biomass (green diamond), glucose concentration (black triangle), and itaconic acid production (red circle) in transformant V08 (filled symbols) and the WT (open symbols)

the wild-type (Fig. 5; [20]). Moreover, compared with the parental strain, V08 showed improved cell growth, glucose consumption and itaconic acid production (Fig. 6). The trend exhibited in our study is similar to those published by Lin et al., where $\mathrm{VHb}$ was expressed in A. terreus under the control of PgpdA [20]. Although the oxygen uptake rate was not that high as reported (Fig. 6a) by Lin et al. [20], the $v g b$ transformant V08 showed faster glucose consumption and itaconic acid production when oxygen availability became limited (after 48 h) (Fig. 6b). The different results might result from the different initial glucose concentrations [160 (our study) vs. $100 \mathrm{~g} / \mathrm{L}]$ or A. terreus hosts [A. terreus CICC 40205 (our study) vs. NRRL 1960] or promoters [PgpdAt3 (our study) vs. PgpdA] or integration sites (the integration into the genome was expected to be random). As reported for A. niger harbouring the $v g b$ gene [32], cell growth reflected by biomass was not greatly affected by the expressed VHb (Fig. 6b). These results prove that the $v g b$ gene was successfully expressed in A. terreus under the control of PgpdAt3 and that PgpdAt was suitable as the promoter for genetic engineering of $A$. terreus.

In summary, we have cloned and functionally characterized the promoter region of the gpd gene from A.terreus.
The PgpdAt promoters with different lengths were able to successfully drive $s g f p$ expression in A. terreus. The shortest one, PgpdAt3 (630 bp), was also successfully applied in genetic engineering of $A$. terreus by driving $v g b$ expression. We therefore have developed an effective and compact native promoter for engineering the important industrial microorganism-A. terreus.

Acknowledgments We are grateful to Chinese Academy of Sciences for the financial support.

\section{References}

1. Lubertozzi D, Keasling JD (2009) Developing Aspergillus as a host for heterologous expression. Biotechnol Adv 27:53-75

2. Klement T, Buchs J (2013) Itaconic acid-a biotechnological process in change. Bioresour Technol 135:422-431

3. Lai LST, Hung CS, Lo CC (2007) Effects of lactose and glucose on production of itaconic acid and lovastatin by Aspergillus terreus ATCC 20542. J Biosci Bioeng 104(1):9-13

4. Meyer V (2008) Genetic engineering of filamentous fungi-progress obstacles and future trends. Biotechnol Adv 26:177-185

5. Lubertozzi D, Keasling JD (2006) Marker and promoter effects on heterologous expression in Aspergillus nidulans. Appl Microbiol Biotechnol 72:1014-1023

6. Ganzlin M, Rinas U (2008) In-depth analysis of the Aspergillus niger glucoamylase ( $g l a A$ ) promoter performance using highthroughput screening and controlled bioreactor cultivation techniques. J Biotechnol 135:266-271

7. Kanemori Y, Gomi K, Kitamoto K, Kumagai C, Tamura G (1999) Insertion analysis of putative functional elements in the promoter region of the Aspergillus oryzae Taka-amylase A gene (amyB) using a heterologous Aspergillus nidulans amdS-lacZ fusion gene system. Biosci Biotechnol Biochem 63:180-183

8. Roth AH, Dersch P (2010) A novel expression system for intracellular production and purification of recombinant affinitytagged proteins in Aspergillus niger. Appl Microbiol Biotechnol 86:659-670

9. Cao Y, Jiao R, Xia Y (2012) A strong promoter PMagpd provides a tool for high gene expression in entomopathogenic fungus Metarhizium acridum. Biotechnol Lett 34:557-562

10. Kim JG, Choi YD, Chang YJ, Kim SU (2003) Genetic transformation of Monascus purpureus DSM1379. Biotechnol Lett 25:1509-1514

11. Punt PJ, Dingemanse MA, Kuyvenhoven A, Soede RD, Pouwels PH, van den Hondel CA (1990) Functional elements in the promoter region of the Aspergillus nidulans gpdA gene encoding glyceraldehyde-3-phosphate dehydrogenase. Gene 93:101-109

12. Van Bogaert IN, De Maeseneire SL, Develter D, Soetaert W, Vandamme EJ (2008) Cloning and characterisation of the glyceraldehyde 3-phosphate dehydrogenase gene of Candida bombicola and use of its promoter. J Ind Microbiol Biotechnol 35:1085-1092

13. Blumhoff M, Steiger MG, Marx H, Mattanovich D, Sauer M (2013) Six novel constitutive promoters for metabolic engineering of Aspergillus niger. Appl Microbiol Biotechnol 97:259-267

14. Dave K, Punekar NS (2011) Utility of Aspergillus niger citrate synthase promoter for heterologous expression. J Biotechnol 155:173-177

15. Bando H, Hisada H, Ishida H, Hata Y, Katakura Y, Kondo A (2011) Isolation of a novel promoter for efficient protein expression by Aspergillus oryzae in solid-state culture. Appl Microbiol Biotechnol 92:561-569 
16. Fleissner A, Dersch P (2010) Expression and export: recombinant protein production systems for Aspergillus. Appl Microbiol Biotechnol 87:1255-1270

17. Liao XG, Fang WG, Zhang YJ, Fan YH, Wu XW, Zhou Q, Pei Y (2008) Characterization of a highly active promoter PBbgpd in Beauveria bassiana. Curr Microbiol 57:121-126

18. Kuo CY, Chou SY, Huang CT (2004) Cloning of glyceraldehyde3-phosphate dehydrogenase gene and use of the gpd promoter for transformation in Flammulina velutipes. Appl Microbiol Biotechnol 65:593-599

19. Tevz G, Bencina M, Legisa M (2010) Enhancing itaconic acid production by Aspergillus terreus. Appl Microbiol Biotechnol 87:1657-1664

20. Lin YH, Li YF, Huang MC, Tsai YC (2004) Intracellular expression of Vitreoscilla hemoglobin in Aspergillus terreus to alleviate effect of a short break in aeration during culture. Biotechnol Lett 26:1067-1072

21. Villanueva A, Maccabe AP, Buesa J, Ramon D (1999) Apparent mRNA instability in Aspergillus nidulans and Aspergillus terreus of a heterologous cDNA encoding the major capsid antigen of Rotavirus. Rev Iberoam Micol 16:130-135

22. Li A, van Luijk N, ter Beek M, Caspers M, Punt P, van der Werf M (2011) A clone-based transcriptomics approach for the identification of genes relevant for itaconic acid production in Aspergillus. Fungal Genet Biol 48:602-611

23. Wen Y, Song Y, Li JL (2001) The effects of Vitreoscilla hemoglobin expression on growth and antibiotic production in Streptomyces cinnamonensis. Chin J Biotechnol 17:24-28

24. Sambrook J, Fritsch EF, Maniatis T (1989) Molecular cloning a laboratory manual, 3rd edn. Cold Spring Harbor Laboratory Press, New York
25. Song P, Cai C, Skokut M, Kosegi B, Petolino J (2002) Quantitative real-time PCR as a screening tool for estimating transgene copy number in WHISKERS-derived transgenic maize. Plant Cell Rep 20:948-954

26. Livak KJ, Schmittgen TD (2001) Analysis of relative gene expression data using real-time quantitative PCR and the $2^{-\Delta \Delta C T}$ Method. Methods 25:402-408

27. Li A, Pfelzer N, Zuijderwijk R, Brickwedde A, van Zeijl C, Punt $\mathrm{P}$ (2013) Reduced by-product formation and modified oxygen availability improve itaconic acid production in Aspergillus niger. Appl Microbiol Biotechnol 97:3901-3911

28. Punt PJ, Kramer C, Kuyvenhoven A, Pouwels PH, van den Hondel CA (1992) An upstream activating sequence from the Aspergillus nidulans gpdA gene. Gene 120:67-73

29. Hirano T, Sato T, Yaegashi K, Enei H (2000) Efficient transformation of the edible basidiomycete Lentinus edodes with a vector using a glyceraldehyde-3-phosphate dehydrogenase promoter to hygromycin B resistance. Mol Gen Genet 263:1047-1052

30. Wei XX, Chen GQ (2008) Applications of the VHb gene $v g b$ for improved microbial fermentation processes. Methods Enzymol 436:273-287

31. Zhang L, Li Y, Wang Z, Xia Y, Chen W, Tang K (2007) Recent developments and future prospects of Vitreoscilla hemoglobin application in metabolic engineering. Biotechnol Adv 25:123-136

32. Hofmann G, Diano A, Nielsen J (2009) Recombinant bacterial hemoglobin alters metabolism of Aspergillus niger. Metab Eng 11:8-12 